\title{
Systemic high-dose methotrexate plus ifosfamide is highly effective for central nervous system (CNS) involvement of lymphoma
}

\author{
Lars Fischer • Agnieszka Korfel • Philipp Kiewe • \\ Martin Neumann • Kristoph Jahnke • Eckhard Thiel
}

Received: 25 January 2008 / Accepted: 21 July 2008 / Published online: 5 August 2008

(C) Springer-Verlag 2008

\begin{abstract}
Patients with malignant central nervous system (CNS) involvement of lymphoma have a poor prognosis with intrathecal chemotherapy and radiation. In this paper, we report the results we obtained in such patients by intravenous chemotherapy with high-dose methotrexate and ifosfamide (HDMTX/IFO). The study involved a review of all patients who received HDMTX/IFO for CNS involvement of malignant lymphoma at our hospital. Therapy consisted of $4 \mathrm{~g} / \mathrm{m}^{2}$ of MTX ( $4 \mathrm{~h}$ infusion on day 1) and $1.5-2 \mathrm{~g} / \mathrm{m}^{2} /$ day of IFO ( $3 \mathrm{~h}$ infusion on days $3-5$ ). The study included 20 patients with a median age of 65 years (range, 30-83) and CNS relapse of a malignant lymphoma. Seventeen patients had been pretreated with up to two chemotherapy regimens. The objective response rate was $90 \%$ with 12 complete or unconfirmed complete (CR and $\mathrm{CRu}$ ) and six partial remissions. All patients had at least stabilization of their neurological symptoms. Myelosuppression was the most common toxicity. The median follow-up time was 14.9 months. The median time to neurological progression was 8.9 months. Twelve patients received subsequent therapy, including high-dose chemotherapy with autologous stem cell transplantation in five cases. The median overall survival was not reached. Systemic chemotherapy with HDMTX/IFO is a feasible and promising treatment modality for CNS relapse of a malignant lymphoma.
\end{abstract}

L. Fischer $(\bowtie) \cdot$ A. Korfel $\cdot$ P. Kiewe $\cdot$ M. Neumann $\cdot$ K. Jahnke

E. Thiel

Department of Hematology, Oncology \& Transfusion Medicine, Charité Universitätsmedizin Berlin, Campus Benjamin Franklin, Hindenburgdamm 30,

12200 Berlin, Germany

e-mail: lars.fischer@charite.de
Keywords Neoplastic meningitis · CNS lymphoma . Ifosfamide $\cdot$ Methotrexate

\section{Introduction}

Central nervous system (CNS) involvement is a serious complication of hematologic malignancies. Standard therapy has not been established for these patients. Patients with leptomeningeal involvement usually receive intrathecal (IT) chemotherapy consisting of methotrexate (MTX) or cytarabine, which is sometimes combined with radiotherapy for nodular tumor masses [1-4]. Patients presenting with parenchymal brain involvement are usually submitted to whole-brain irradiation (WBI). With this therapy, patients with malignant CNS involvement generally have a poor prognosis with a median survival of usually less than 6 months. Systemic disease is a frequent cause of death in these patients [5-8].

In the management of CNS malignancies, systemic therapy offers several potential advantages over local treatment modalities like IT chemotherapy or radiation, the most important one being simultaneous treatment of the systemic disease [5, 8, 9]. Furthermore, systemic application achieves a more uniform distribution of the drug throughout the neuroaxis and obviates ventricular reservoir insertion or multiple lumbar punctures (LP) for drug instillation with all the potential acute and late complications. Finally, a much wider array of chemotherapeutic agents can be administered by intravenous (IV) than by IT application. On the other hand, considerable systemic toxicity is associated with IV chemotherapy, whereas IT therapy poses potential infectious and neurotoxic risks. 
Both systemic ifosfamide and high-dose MTX (doses of $1.0 \mathrm{~g} / \mathrm{m}^{2}$ or more) reach cytostatic levels in the cerebrospinal fluid (CSF) and are effective for treating various malignancies [10-12]. This monocenter study retrospectively analyzed the activity and toxicity of combination chemotherapy with high-dose methotrexate and ifosfamide (HDMTX/IFO) for CNS manifestations of non-Hodgkin's lymphoma.

\section{Materials and methods}

\section{Patients}

The study included all patients treated with HDMTX/IFO for CNS lymphoma (including the retina) at our institution from July 2002 to August 2007. CNS disease was diagnosed radiologically [brain lesions and/or meningeal enhancement on cranial and spinal magnetic resonance imaging (MRI) or computed tomography (CT)] or by CSF cytomorphology or immunocytology. Intraocular malignancy was diagnosed by slit-lamp examination and aqueous cytology or chorioretinal biopsy. All patients underwent a staging procedure for systemic tumor manifestations with bone marrow biopsy and contrast-enhanced $\mathrm{CT}$ of the thorax and abdomen.

Exclusion criteria were creatinine clearance $<50 \mathrm{ml} / \mathrm{min}$, liver disease with elevation of bilirubin $>2 \mathrm{mg} / \mathrm{dl}$, previous WBI, and active systemic infection. All patients gave written informed consent before starting treatment.

\section{Treatment}

Patients received an IV infusion of MTX $4 \mathrm{~g} / \mathrm{m}^{2}$ over $4 \mathrm{~h}$ on day 1 and IFO 1.5 or $2 \mathrm{~g} / \mathrm{m}^{2} /$ day over $3 \mathrm{~h}$ on days 3 to 5 . The MTX dose was adjusted for creatinine clearance (CC) $<100 \mathrm{ml} / \mathrm{min}$ as follows: $\mathrm{CC} / 100 \times 4 \mathrm{~g} / \mathrm{m}^{2}$. Supportive therapy included antiemetics, intravenous hydration, urine alkalization, mesna, and a leucovorin rescue beginning $24 \mathrm{~h}$ after the start of MTX infusion. Systemic corticosteroids were applied only for symptom control at the beginning of therapy. Treatment was continued for a maximum of eight cycles and stopped in cases where further therapy was prevented by radiologic or CSF progression, clinical deterioration, or toxicity.

\section{Response and toxicity}

In patients treated after August 2005, response of parenchymal lesions was evaluated according to the then published criteria of the International Primary CNS Lymphoma Collaborative Group (IPCG) [13]. Those included the specification of an unconfirmed complete remission $(\mathrm{CRu})$ for cases with small but persisting enhancing abnormalities on brain imaging or patients fulfilling the criteria for complete remission (CR) but still receiving corticosteroids. In three patients treated before 2005, IPCG guidelines were used for a retrospective evaluation of parenchymal brain lesions. These patients had CR according to both Cheson criteria [14] used initially and IPCG criteria used for the present analysis.

In leptomeningeal disease, $\mathrm{CR}$ was defined as a negative CSF cytomorphology in two consecutive LP and disappearance of meningeal enhancement on gadolinium MRI, if present at first presentation. Progressive disease (PD) was defined as an increase of CSF tumor cells, while stable disease (SD) was considered to be any other condition. Response and SD were only documented if neurological symptoms had improved or remained stable; patients were otherwise assessed as having PD [2, 4]. Response of measurable systemic disease was documented according to Cheson's criteria [14].

Neurological response was classified as complete response, improvement, stable disease, or worsening. Neurological complete response was defined as complete resolution of all neurological symptoms and signs attributable to the malignant disease, improvement as regression of symptoms with residual deficits, worsening as neurological deterioration, and stable disease as no improvement in the absence of new symptoms.

Response evaluation of all CNS tumor sites using the initial method (MRI, CT, CSF cytomorphology/immunocytology, and ophthalmologic examination) was performed at least after every second chemotherapy cycle and every 3 months after therapy. A full clinical and neurological assessment, including toxicity evaluation, was performed before each chemotherapy course.

Adverse events were graded according to the Common Terminology Criteria (CTC) of the National Cancer Institute, version 3.0, and the highest observed grade was recorded for each patient.

\section{Statistical analysis}

A descriptive analysis of qualitative and quantitative variables was done. Frequencies, median values, and ranges were specified when applicable. Estimates of the overall survival (OAS) and time to neurological progression (TTP) were calculated for all patients using the Kaplan-Meier method. The OAS was calculated from the start of therapy to the last follow-up or to death from any cause. The time to neurological progression was measured from the start of therapy until neurological deterioration, death from CNS disease, or the last follow-up. Responding or stable patients who continued with other treatment (due to toxicity or the attending physician's decision) were censored for calculation of the TTP. 


\section{Results}

\section{Patient characteristics}

Twenty patients with a median age of 65 years (Table 1) were treated with at least one HDMTX/IFO course. Meningeal involvement was confirmed by cytomorphologic CSF evaluation in all patients. Parenchymal brain involvement was confirmed by biopsy in all but two patients who had developed parenchymal brain lesions during treatment for histologically proven systemic lymphoma. Seventeen patients had diffused large B-cell lymphoma (DLBCL) in the CNS. Secondary CNS involvement of systemic DLBCL was present in eight of them and a relapsed PCNSL/PIOL in seven, while two had an aggressive large-cell lymphoma in the CNS in the presence of systemic indolent B-cell lymphoma (Richter's transformation of Waldenstrom's macroglobulinemia). Two patients had simultaneous systemic and CNS involvement of mantle cell lymphoma and

Table 1 Patient characteristics at diagnosis of CNS disease

\begin{tabular}{|c|c|c|}
\hline \multirow[t]{2}{*}{ Characteristics } & \multicolumn{2}{|c|}{ Values } \\
\hline & $n$ & Percent \\
\hline Age (years) & \multicolumn{2}{|c|}{$30-83$ (median 65) } \\
\hline Age $>60$ & \multicolumn{2}{|c|}{14} \\
\hline Male/female & \multicolumn{2}{|c|}{$10 / 10$} \\
\hline \multicolumn{3}{|l|}{ Histologic diagnosis (CNS) } \\
\hline DLBCL & 17 & 85 \\
\hline Mantle cell lymphoma & 1 & 5 \\
\hline Marginal zone lymphoma & 1 & 5 \\
\hline T-cell lymphoma & 1 & 5 \\
\hline \multicolumn{3}{|l|}{ CNS involvement } \\
\hline Meningeal & 3 & 15 \\
\hline Parenchymal & 13 & 65 \\
\hline Meningeal+parenchymal & 3 & 15 \\
\hline Ocular & 1 & 5 \\
\hline Systemic disease & 9 & 45 \\
\hline Primary CNS involvement /CNS relapse & $3 / 17$ & $15 / 85$ \\
\hline \multicolumn{3}{|l|}{ Previous therapy } \\
\hline 1 regimen & 13 & 65 \\
\hline 2 regimens & 4 & 20 \\
\hline \multicolumn{3}{|l|}{ Previous CNS directed therapy } \\
\hline 1 regimen & 9 & 45 \\
\hline 2 regimens & 1 & 5 \\
\hline \multicolumn{3}{|l|}{ Symptoms ${ }^{\mathrm{a}}$} \\
\hline Cranial nerve palsy & 5 & 25 \\
\hline Sensorimotor deficits & 4 & 20 \\
\hline Nausea/Emesis & 4 & 20 \\
\hline Pain & 4 & 20 \\
\hline Cerebellar & 5 & 20 \\
\hline Neuropsychological & 5 & 20 \\
\hline Other & 11 & 55 \\
\hline
\end{tabular}

${ }^{a}$ More than one symptom per patient possible marginal zone lymphoma, respectively, and one patient had a T-cell lymphoma.

Three patients with systemic indolent B-cell lymphoma already had CNS disease at first presentation and were therapy-naive. All others had a CNS relapse and were pretreated with up to two different chemotherapy regimens. Systemic pretreatment regimens were CHOP-like (with or without rituximab). CNS-directed pretreatment (one or two therapy lines) had been given to ten patients and consisted of IT chemotherapy with MTX or IV chemotherapy with HDMTX, high-dose cytarabine, ifosfamide, or topotecan. Nine patients $(45 \%)$ had concomitant systemic disease.

\section{Therapy}

Patients were treated with a median of five HDMTX/IFO cycles (range, 1 to 8 ). Nine patients received the full MTX dose, while 11 had a dose adjustment to $50-90 \%$ based on their creatinine clearance. Dose adjustment was necessary in nine $(64 \%)$ patients $>60$ years but only in two $(33 \%)$ younger ones $(p=0.28)$.

HDMTX/IFO was terminated prematurely in ten patients for the following reasons: creatinine clearance $<50 \mathrm{ml} / \mathrm{min}$ ( $n=3$, all with grade 2 nephrotoxicity), the doctor's decision to intensify treatment by high-dose chemotherapy with autologous stem cell transplantation (SCT; $n=3$ ), disease progression $(n=2)$, the patient's request $(n=1)$, and toxic death $(n=1)$. In three other patients, therapy was modified as follows: An individual decision was made to stop HDMTX after one to five cycles due to grade 1,2 , or 3 nephrotoxicity, and therapy was continued with IFO alone. Therapy had to be modified or terminated due to toxicity or renal function impairment in five $(36 \%)$ patients over 60 and in one $(25 \%)$ younger patient $(p=0.4)$.

No concurrent lymphoma-directed therapy was given except to one patient with CNS relapse of a mantle cell lymphoma, who was additionally treated with IT liposomal cytarabine.

Granulocyte colony-stimulating factor (G-CSF)-supported mobilization of peripheral blood stem cells after HDMTX/ IFO in six patients was successful in all cases. Five of them proceeded to high-dose chemotherapy with carmustine or melphalan in combination with thiotepa followed by autologous stem cell transplantation (three for consolidation and two at relapse), and eight patients received various other consolidation or salvage treatments (systemic therapy with topotecan, high-dose cytarabine or zevaline, WBI, or IT chemotherapy).

\section{Response}

Eighteen patients (90\%) responded to HDMTX/IFO with a $\mathrm{CR}$ in ten cases, a $\mathrm{CRu}$ in two, and a PR in six (Fig. 1). 
Fig. 1 Patient with $\mathrm{CNS}$ and pulmonary relapse of DLBCL responding to therapy (a-c cranial MRI, $\mathbf{d}-\mathbf{e}$ chest $\mathrm{CT}$ scan): a, d after partial surgical removal of CNS tumor and before chemotherapy; b, d after four courses of HDMTX/IFO; $\mathbf{c}$ 3 months after high-dose carmustine/thiotepa and autologous SCT
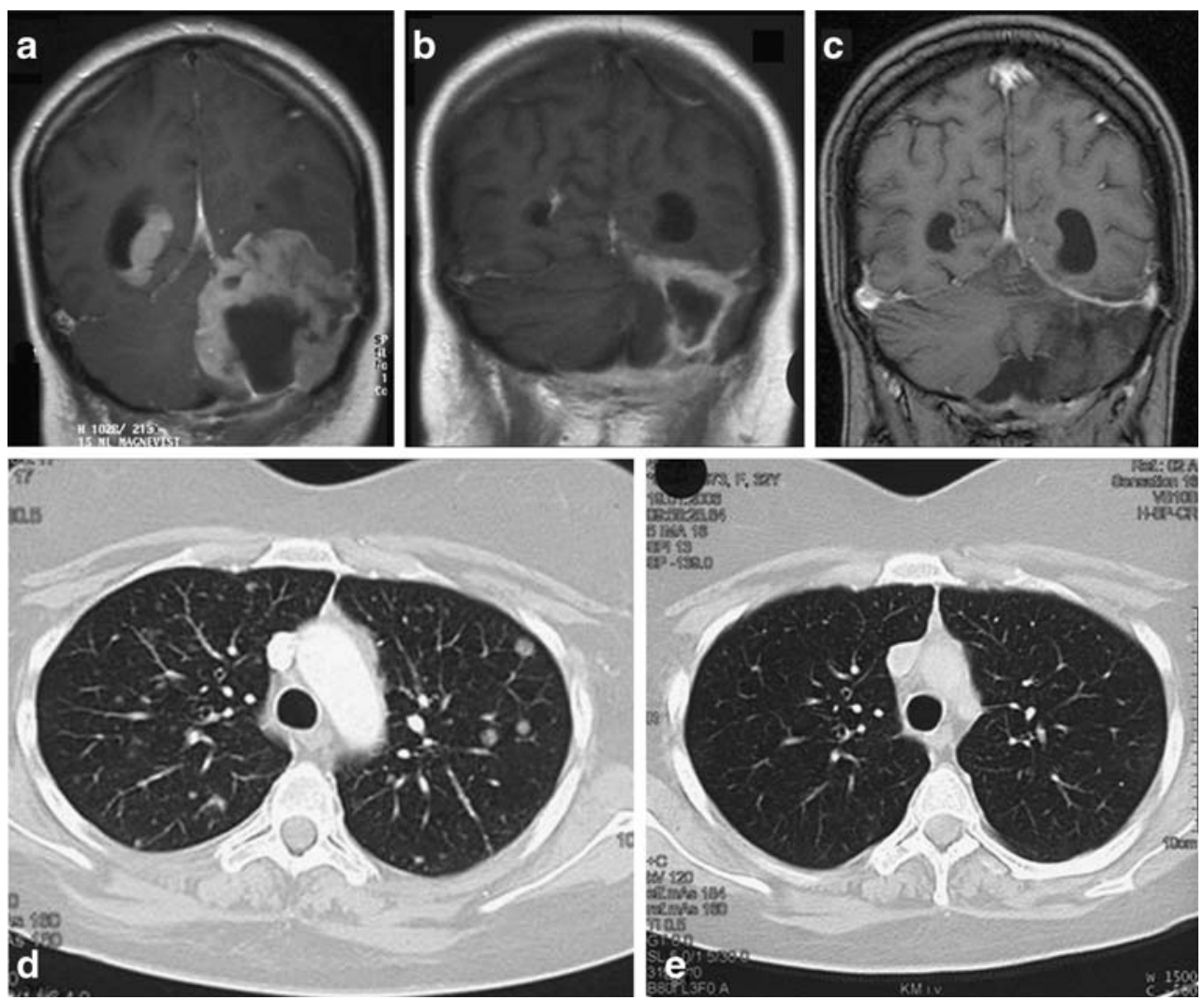

Two patients had SD for 1.9 and 5.1 months. Neurological symptoms resolved completely in eight patients, improved in nine, and remained stable over 1.3 to 5.5 months in three (two with an objective response and one with stable CNS disease).

Pretreatment with HDMTX led to a CNS response rate of $85.7 \%$ (six of seven patients) as compared to $92.3 \%$ (12 of 13 patients) with other regimens or without pretreatment $(p=0.6)$.

Systemic manifestations responded in four patients: axillary lymph node enlargement in a thyroid non-Hodgkin's lymphoma disappeared completely, as did intrapulmonary nodules in a relapsed PCNSL and bone marrow infiltration in a marginal zone lymphoma after six to eight cycles of HDMTX/IFO, while pulmonary manifestations in one systemic DLBCL were in partial remission after four cycles.

One patient with Waldenstrom's macroglobulinemia remained stable according to his paraprotein levels. In four patients, systemic disease (bone marrow involvement of DLBCL or indolent lymphoma) was not reevaluated due either to the doctor's decision or to death before systemic response evaluation.

\section{Toxicity}

Myelosuppression was the most frequent toxicity and involved grades 3 and 4 leukopenia in 13 patients $(65 \%)$, grade 3 and 4 thrombopenia in six (30\%), and grade 3 anemia in four $(20 \%)$. There were no significant differences in the frequency of thrombopenia and anemia between patients over 60 years and younger ones. However, grade 4 leukopenia occurred only in patients over 60 . Grades 3, 2, and 1 infection was observed in one patient each. Grade 3 nephrotoxicity occurred in one patient and grades 1 and 2 in 11 patients, leading to omission of HDMTX in three of them. In three patients, HDMTX/IFO was terminated prematurely due to nephrotoxicity grade 2 . One patient developed acute ifosfamide-related grade 2 encephalopathy, which reversed spontaneously after 3 days. Other toxicities were nausea (grade 3 in one patient and grades 1 and 2 in nine patients) and fatigue (grade 3 in one patient and grades 1 and 2 in seven patients).

There were two therapy-related deaths, both due to sepsis in neutropenia: An 83-year-old patient with DLBCL and bone marrow infiltration died after two chemotherapy cycles, and a 73-year-old patient with stage IV mantle cell lymphoma died after six chemotherapy courses.

\section{Survival}

After a median follow-up of 14.9 months, 13 patients were alive, and seven had died (five from tumor progression and two of toxicity). The site of relapse/progression was the CNS in all patients. For the whole patient population, the median TTP was 8.9 months (range 1.3 to 30 months), and 
the median OAS was not reached (range 1.8 to $50+$ months; Fig. 2).

There were no significant differences in outcome with respect to age ( $\leq 60$ versus $>60$ years), meningeal involvement, or lymphoma type (Table 2); however, a trend toward longer survival was seen in patients without meningeal involvement and with PCNSL. The estimated survival at 12 months was $55 \%$ for older and $83 \%$ for younger patients $(p=0.2)$.

Seven patients survived longer than 12 months; four of them received high-dose therapy with autologous stem-cell transplantation after HDMTX/IFO.

\section{Discussion}

Data supporting the utility of systemic chemotherapy for secondary CNS involvement in malignant disease are mostly limited to small phase II studies or retrospective analyses of heterogeneous patient populations. Some analyses suggested a survival advantage for CNS-directed systemic chemotherapy and found no evidence for a superiority of IT therapy $[1,15-17]$.

Two studies have reported on systemic HDMTX monotherapy for CNS manifestations of lymphoma and solid tumors $[18,19]$. Glantz et al. found that treatment with $8 \mathrm{~g} / \mathrm{m}^{2}$ of MTX led to a high-objective response rate of $81 \%$ and a median survival of 13.8 months in 16 patients with lymphoma and solid tumor neoplastic meningitis. The study suggested a superiority of systemic HDMTX over IT MTX applied in a historic control group [18]. Lassmann et al.

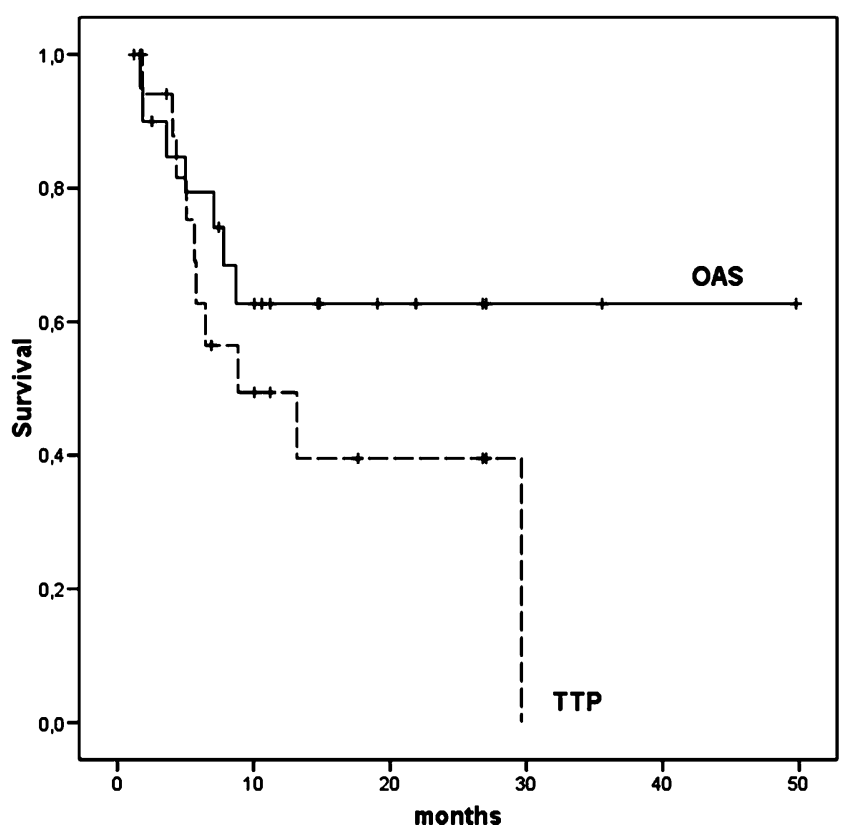

Fig. 2 Overall survival $(O A S)$ and time to neurological progression (TTP) for all patients
Table 2 Outcome by age, meningeal involvement, and lymphoma type

\begin{tabular}{lll}
\hline & $\begin{array}{l}\text { Median TTP } \\
\text { (months) }\end{array}$ & $\begin{array}{l}\text { Median OAS } \\
\text { (months) }\end{array}$ \\
\hline Age & & \\
$\leq 60$ years & n.r. & n.r. \\
$>60$ years & 6.5 & n.r. \\
& $p=0.1$ & $p=0.2$ \\
Meningeal involvement: & & \\
no (n=14) & 8.9 & n.r. \\
yes (n=6) & 5.8 & 7.1 \\
& $p=1.0$ & $p=0.066$ \\
Diagnosis: & & \\
PCNSL (n=7) & 8.9 & n.r. \\
Secondary CNS lymphoma (n=13) & 13.1 & 8.7 \\
& $p=0.9$ & $p=0.08$ \\
\hline
\end{tabular}

n.r. Not reached

administered $3.5 \mathrm{~g} / \mathrm{m}^{2}$ of MTX to 23 solid tumor patients, $44 \%$ of whom had isolated meningeal metastases, and reported a PR in $33 \%$ and a median OAS of 20 weeks [19]. In a prospective study, the combination of $3.5 \mathrm{~g} / \mathrm{m}^{2}$ MTX, procarbazine, and IT cytarabine in 23 patients with secondary CNS lymphoma achieved a response rate of $100 \%$ with a 33\% CR rate for leptomeningeal disease. The median OAS of the whole group was 6 months [5].

Little data is available on IFO for treatment of malignant CNS disease. The drug was included in treatment protocols that proved effective in PCNSL and intraocular lymphoma, both at first diagnosis and at relapse [20-22].

The present study included a relatively old population with a high proportion of pretreated and potentially chemotherapy-resistant patients. An age of over 60 is a well-established risk factor in both PCNSL and systemic lymphoma $[23,24]$. Our study nevertheless achieved a high objective response rate of $90 \%$. Moreover, the median TTP of 8.9 months compares favorably to the outcome reported in the literature. The study regimen proved effective for both parenchymal and meningeal involvement as well as concomitant systemic disease. Remarkably, a response was observed in nine of ten patients pretreated with up to two CNS-directed therapies, including HDMTX in six patients. Survival tended to be shorter in patients with meningeal disease than in those with brain parenchyma involvement. The reason for this difference remains speculative. These patient groups were comparable for age and pretreatment; however, four of six patients with meningeal disease had secondary CNS lymphoma. It may be speculated whether secondary CNS lymphoma has a generally worse prognosis than PCNSL.

Our results should be interpreted with caution because of some obvious study limitations such as the retrospective analysis and the relatively small number of patients. 
Moreover, our patient population was heterogeneous in terms of lymphoma subtype (including relapsed systemic DLBCL, PCNSL, and indolent CNS lymphoma), pretreatment (three patients were therapy-naive), and post-study treatment. In particular, subsequent treatment with highdose chemotherapy and autologous stem cell transplantation in five patients might have contributed to the favorable overall survival. High-dose chemotherapy with stem-cell transplantation is the only treatment associated with prolonged survival in patients with CNS involvement of aggressive lymphomas $[25,26]$.

WBI alone may represent an effective salvage treatment for many PCNSL patients [27]. However, there is growing evidence linking it to delayed neurotoxicity in these cases [28-30]. On the other hand, different salvage chemotherapy regimens have proven effective for PCNSL [31]. Thus, delaying WBI in relapsed PCNSL seems a reasonable approach. Since the risk of late neurotoxicity appears to be particularly high when HDMTX is given after WBI [32], patients pretreated with WBI do not receive HDMTX at our institution and were therefore excluded from this analysis. This might have introduced a selection bias, since relapse after WBI could entail a different prognosis than relapse after chemotherapy alone.

HDMTX/IFO had considerable hematotoxicity with a $70 \%$ incidence of CTC grades 3 and 4 leukopenia and an 8.7\% treatment-related mortality rate. Prophylactic administration of G-CSF should therefore be considered in this protocol. Antibacterial and, in patients receiving corticosteroids, possibly antifungal prophylaxis might also reduce the rate of infectious complications, especially in older patients. Both HDMTX and IFO are potentially nephrotoxic, but renal function impairment was generally manageable with dose adjustment according to creatinine clearance. However, therapy had to be modified and terminated prematurely due to renal insufficiency in $30 \%$ of patients.

Systemic HDMTX/IFO seems to be a promising therapy option for CNS involvement of lymphoma. Our results justify evaluation of the MTX/IFO protocol in a prospective study.

\section{References}

1. Bokstein F, Lossos A, Siegal T (1998) Leptomeningeal metastases from solid tumors: a comparison of two prospective series treated with and without intra-cerebrospinal fluid chemotherapy. Cancer 82:1756-1763. doi:10.1002/(SICI)1097-0142(19980501) 82:9<1764::AID-CNCR24>3.0.CO;2-1

2. Glantz MJ, LaFollette S, Jaeckle KA et al (1999) Randomized trial of a slow-release versus a standard formulation of cytarabine for the intrathecal treatment of lymphomatous meningitis. J Clin Oncol 17:3110-3116

3. Glantz MJ, Jaeckle KA, Chamberlain MC et al (1999) A randomized controlled trial comparing intrathecal sustained- release cytarabine (DepoCyt) to intrathecal methotrexate in patients with neoplastic meningitis from solid tumors. Clin Cancer Res 5:3394-3402

4. Grossman SA, Finkelstein DM, Ruckdeschel JC et al (1993) Randomized prospective comparison of intraventricular methotrexate and thiotepa in patients with previously untreated neoplastic meningitis. Eastern Cooperative Oncology Group. J Clin Oncol 11:561-569

5. Bokstein F, Lossos A, Lossos IS et al (2002) Central nervous system relapse of systemic non-Hodgkin's lymphoma: results of treatment based on high-dose methotrexate combination chemotherapy. Leuk Lymphoma 43:587-593. doi:10.1080/1042819 0290012092

6. Bollen EL, Brouwer RE, Hamers S et al (1997) Central nervous system relapse in non-Hodgkin lymphoma. A single-center study of 532 patients. Arch Neurol 54:854-859

7. Lagerwaard FJ, Levendag PC, Nowak PJ et al (1999) Identification of prognostic factors in patients with brain metastases: a review of 1292 patients. Int J Radiat Oncol Biol Phys 43:795803. doi:10.1016/S0360-3016(98)00442-8

8. van Besien K, Ha CS, Murphy S et al (1998) Risk factors, treatment, and outcome of central nervous system recurrence in adults with intermediate-grade and immunoblastic lymphoma. Blood 91:1178-1184

9. Blaney SM, Poplack DG (1996) Pharmacologic strategies for the treatment of meningeal malignancy. Invest New Drugs 14:69-85. doi:10.1007/BF00173684

10. Hiraga S, Arita N, Ohnishi T et al (1999) Rapid infusion of highdose methotrexate resulting in enhanced penetration into cerebrospinal fluid and intensified tumor response in primary central nervous system lymphomas. J Neurosurg 91:221-230

11. Tetef ML, Margolin KA, Doroshow JH et al (2000) Pharmacokinetics and toxicity of high-dose intravenous methotrexate in the treatment of leptomeningeal carcinomatosis. Cancer Chemother Pharmacol 46:19-26. doi:10.1007/s002800000118

12. Yule SM, Price L, Pearson AD et al (1997) Cyclophosphamide and ifosfamide metabolites in the cerebrospinal fluid of children. Clin Cancer Res 3:1985-1992

13. Abrey LE, Batchelor TT, Ferreri AJ et al (2005) Report of an international workshop to standardize baseline evaluation and response criteria for primary CNS lymphoma. J Clin Oncol 23:5034-5043. doi:10.1200/JCO.2005.13.524

14. Cheson BD, Horning SJ, Coiffier B et al (1999) Report of an international workshop to standardize response criteria for nonHodgkin's lymphomas. NCI Sponsored International Working Group. J Clin Oncol 17:1244

15. Boogerd W, Hart AA, van der Sande JJ et al (1991) Meningeal carcinomatosis in breast cancer. Prognostic factors and influence of treatment. Cancer 67:1685-1695. doi:10.1002/1097-0142 (19910315)67:6<1685::AID-CNCR2820670635>3.0.CO;2-M

16. Herrlinger U, Forschler H, Kuker W et al (2004) Leptomeningeal metastasis: survival and prognostic factors in 155 patients. J Neurol Sci 223:167-178. doi:10.1016/j.jns.2004.05.008

17. Siegal T, Lossos A, Pfeffer MR (1994) Leptomeningeal metastases: analysis of 31 patients with sustained off-therapy response following combined-modality therapy. Neurology 44:1463-1469

18. Glantz MJ, Cole BF, Recht L et al (1998) High-dose intravenous methotrexate for patients with nonleukemic leptomeningeal cancer: is intrathecal chemotherapy necessary? J Clin Oncol $16: 1561-1567$

19. Lassman AB, Abrey LE, Shah GD et al (2006) Systemic highdose intravenous methotrexate for central nervous system metastases. J Neurooncol 78:255-260. doi:10.1007/s11060-005-9044-6

20. Arellano-Rodrigo E, Lopez-Guillermo A, Bessell EM et al (2003) Salvage treatment with etoposide (VP-16), ifosfamide and cytarabine (Ara-C) for patients with recurrent primary central 
nervous system lymphoma. Eur J Haematol 70:219-224. doi:10.1034/j.1600-0609.2003.00045.x

21. Pels H, Schmidt-Wolf IG, Glasmacher A et al (2003) Primary central nervous system lymphoma: results of a pilot and phase II study of systemic and intraventricular chemotherapy with deferred radiotherapy. J Clin Oncol 21:4489-4495. doi:10.1200/JCO.2003.04.056

22. Jahnke K, Wagner T, Bechrakis NE et al (2005) Pharmacokinetics and efficacy of ifosfamide or trofosfamide in patients with intraocular lymphoma. Ann Oncol 16:1974-1978. doi:10.1093/annonc/mdi409

23. Ferreri AJ, Blay JY, Reni M et al (2003) Prognostic scoring system for primary CNS lymphomas: the International Extranodal Lymphoma Study Group experience. J Clin Oncol 21:266-272. doi:10.1200/JCO.2003.09.139

24. Shipp MA (1997) Can we improve upon the international index? Ann Oncol 8(Suppl 1):43-47. doi:10.1023/A:1008245615520

25. Alvarnas JC, Negrin RS, Horning SJ et al (2000) High-dose therapy with hematopoietic cell transplantation for patients with central nervous system involvement by non-Hodgkin's lymphoma. Biol Blood Marrow Transplant 6:352-358. doi:10.1016/ S1083-8791(00)70060-7

26. Jahnke K, Thiel E, Martus P et al (2006) Retrospective study of prognostic factors in non-Hodgkin lymphoma secondarily involving the central nervous system. Ann Hematol 85:45-50. doi:10.1007/ s00277-005-1096-3

27. Hottinger AF, DeAngelis LM, Yahalom J et al (2007) Salvage whole brain radiotherapy for recurrent or refractory primary CNS lymphoma. Neurology 69:1178-1182. doi:10.1212/01.wnl.0000 276986.19602.c1

28. Fischer L, Thiel E, Klasen HA et al (2004) Response of relapsed or refractory primary central nervous system lymphoma (PCNSL) to topotecan. Neurology 62:1885-1887

29. Herrlinger U, Kuker W, Uhl M et al (2005) NOA-03 trial of highdose methotrexate in primary central nervous system lymphoma: final report. Ann Neurol 57:843-847. doi:10.1002/ana.20495

30. Omuro AM, Ben Porat LS, Panageas KS et al (2005) Delayed neurotoxicity in primary central nervous system lymphoma. Arch Neurol 62:1595-1600. doi:10.1001/archneur.62.10.1595

31. Reni M, Ferreri AJ (2001) Therapeutic management of refractory or relapsed primary central nervous system lymphomas. Ann Hematol 80(Suppl 3):B113-B117. doi:10.1007/s002770000230

32. Blay JY, Conroy T, Chevreau C et al (1998) High-dose methotrexate for the treatment of primary cerebral lymphomas: analysis of survival and late neurologic toxicity in a retrospective series. J Clin Oncol 16:864-871 